\title{
PRMT1-RBM15 axis regulates megakaryocytic differentiation of human umbilical cord blood CD34+ cells
}

\author{
SHUILING JIN ${ }^{1 *}$, YANFANG MI ${ }^{2 *}$, JING SONG $^{1}$, PEIPEI ZHANG ${ }^{3}$ and YANYAN LIU ${ }^{3}$ \\ Departments of ${ }^{1}$ Internal Medicine and ${ }^{2}$ Otolaryngology Head and Neck Surgery, \\ The Second Affiliated Hospital of Zhengzhou University, Zhengzhou, Henan 450014; ${ }^{3}$ Department of Internal Medicine, \\ Henan Cancer Hospital and Affiliated Cancer Hospital of Zhengzhou University, Zhengzhou, Henan 450008, P.R. China
}

Received February 15, 2017; Accepted September 13, 2017

DOI: $10.3892 / \mathrm{etm} .2018 .5693$

\begin{abstract}
Protein arginine methyltransferase 1 (PRMT1) serves pivotal roles in various cellular processes. However, its role in megakaryocytic differentiation has not been clearly reported. The aim of the present study was to explore the role of the PRMT-RNA binding motif protein 15 (RBM15) axis in human MK differentiation and the feasibility of targeting PRMT1 for leukemia treatment. In the present study, PRMT1 was overexpressed and the RBM15 protein was knocked down in human umbilical cord blood cluster of differentiation (CD) $34^{+}$cells and the cells were then cultured in megakaryocytic differentiation medium. Flow cytometry was used to analyze CD41 and CD42 double-positive cells, as well as the protein expression levels of PRMT1 and RBM15. The results demonstrated that human cord blood $\mathrm{CD}_{3} 4^{+}$cells differentiate into mature MKs in high thrombopoitin medium, as demonstrated by CD41 and CD42 expression. Overexpression of PRMT1 in human umbilical cord blood CD $34^{+}$cells blocked the maturation of megakaryocytic cells. Knockdown of RBM15 by short hairpin RNA produced less mature MKs. PRMT1 inhibitor rescued PRMT1-blocked megakaryocytic differentiation. These results provide evidence for a novel role of PRMT1 in the negative regulation of megakaryocytic differentiation. PRMT1 may be a therapeutic target for leukemia treatment.
\end{abstract}

Correspondence to: Professor Yanyan Liu, Department of Internal Medicine, Henan Cancer Hospital and Affiliated Cancer Hospital of Zhengzhou University, 127 Dong Ming Road, Zhengzhou, Henan 450008, P.R. China

E-mail: yyliu@zzu.edu.cn

*Contributed equally

Key words: protein arginine methyltransferase 1, RNA binding motif protein 15 , megakaryocytic differentiation, human cord blood, cluster of differentiation $34^{+}$cells

\section{Introduction}

Defective megakaryopoiesis is responsible for various types of blood disorders (1). Therefore, it is regarded as a crucial source for treating thrombocytopenia, acute megakaryocytic leukemia (M7), myeloproliferative diseases and myelodysplastic syndrome (2-4). Understanding the molecular mechanisms of megakaryocytic differentiation is important for developing novel therapies for hematopoietic malignancy with defective MKs. Previous data has demonstrated that protein arginine methyltransferase 1 (PRMT1) has high expression in leukemia, particularly in M7 (5). RNA binding motif protein 15 (RBM15) is required for the long-term maintenance of the homeostasis of hematopoietic stem cells and for MK differentiation (6,7). RBM15 stability is controlled by PRMT1 via CNOT4-mediated ubiquitylation (8). To investigate the biological significance of PRMT1-mediated methylation of RBM15, the present study analyzed the role of the PRMT1-RBM15 axis in megakaryopoiesis. The present study demonstrated that human umbilical cord blood cluster of differentiation (CD) $34^{+}$cells differentiated into mature MKs when they were cultured in high thrombopoitin (TPO) medium. With MK maturation, the PRMT1 protein level decreased, while the RBM15 protein level increased. Overexpression of PRMT1 and knock down of RBM15 blocked the maturation of MKs due to the reduced RBM15 protein level. A PRMT1 inhibitor rescued the PRMT1-blocked megakaryocytic differentiation. These results provide evidence for a novel role of PRMT1 in the negative regulation of megakaryocytic differentiation. Targeting PRMT1 may be a valuable therapeutic method for defective MK diseases.

\section{Materials and methods}

Purification of cord blood CD34 $4^{+}$cells. The present study was approved by the Ethics Committee of The Second Affiliated Hospital of Zhengzhou University (Zhengzhou, China). Human umbilical cord blood was collected from 3 healthy pregnant women aged 28,30 and 33 years old in The Second Affiliated Hospital of Zhengzhou University from October 2015 to April 2016. Informed content was provided from each patient. Following separation with Ficoll gradient as previously mentioned (9), the $\mathrm{CD} 34^{+}$cells were purified with 
anti-CD34 magnetic beads (Miltenyi Biotec GmbH, Bergisch Gladbach, Germany) in accordance with the manufacturer's protocol. Subsequently, the cells were cultured at $37^{\circ} \mathrm{C}$ for $48 \mathrm{~h}$ in Iscove's modified Dulbecco's medium (IMDM; Gibco; Thermo Fisher Scientific, Inc., Waltham, MA, USA) supplemented with $20 \%$ serum substitutes (BIT; Stemcell Technologies, Inc., Vancouver, BC, Canada) and a cytokine mixture $[20 \mathrm{ng} / \mathrm{ml}$ TPO, $20 \mathrm{ng} / \mathrm{ml}$ interleukin-6, $100 \mathrm{ng} / \mathrm{ml}$ stem cell factor (SCF) and $10 \mathrm{ng} / \mathrm{ml}$ Flt3 ligand (PeproTech, Inc., Rocky Hill, NJ, USA)] (10). The purification achieved $95 \%$ purity for CD34 ${ }^{+}$ cells according to fluorescence-activated cell sorting (FACS) analysis. The cells were collected by centrifugation ( $300 \mathrm{x} \mathrm{g}$ at room temperature for $5 \mathrm{~min}$ ), the supernatant was aspirated and the cells were washed twice with iced PBS. The cells were blocked with Human BD Fc Block ${ }^{\mathrm{TM}}$ (cat. no. 564219; BD Biosciences, Franklin Lakes, NJ, USA) at a dilution of 1:100 and incubated at room temperature for $30 \mathrm{~min}$. A total of $1 \mu \mathrm{l}$ anti-CD34 fluorescein isothiocyanate-conjugated antibodies (cat. no. 560238; 1:100; BD Biosciences) was added to $100 \mu \mathrm{l}$ PBS containing 1\% Fetal Bovine Serum (FBS), the cells were cultured in this medium at room temperature for $30 \mathrm{~min}$. Cells were then analyzed using a BD LSRFortessa ${ }^{\mathrm{TM}}$ machine with BD FACSDiva $^{\mathrm{TM}}$ software (version 8.0) (both BD Biosciences) and FlowJo software (version 7.6.5; FlowJo LLC, Ashland, OR, USA). To induce MK differentiation, the expanded CD34 $4^{+}$ cells were cultured at $37^{\circ} \mathrm{C}$ in IMDM medium supplemented with $20 \%$ BIT with cytokine mixture (TPO 100 and $2 \mathrm{ng} / \mathrm{ml}$ SCF). The expression of CD41 and CD42 was analyzed by flow cytometry 10 days after TPO stimulation. To further investigate the function of PRMT1 in megakaryocytic differentiation, a PRMT1 inhibitor DB75 (Sigma-Aldrich; Merck $\mathrm{KGaA}$ ) was used to treat the cells overexpressing PRMT1 protein and cultured in the MK differentiation medium. For DB75 treatment, $20 \mu \mathrm{M}$ DB75 was added to the medium for 10 days, which was every 3 days.

Virus production and transduction. The lentiviral vector, pTripZ (Open Biosystems; Dharmacon), was engineered to express PRMT1 by replacing the shRNA cassette with the PRMT1 coding sequence to achieve robust PRMT1 expression in hematopoietic cells. A total of $15 \mu \mathrm{g}$ lentivirus plasmids expressing the cDNA of PRMT1 V2, short hairpin (sh)RBM15\#1 and shRBM15\#2 $(11,12)$ were transfected into 293T cells (American Type Culture Collection, Manassas, VA, USA) with helper plasmids. The $293 \mathrm{~T}$ cells were cultured in Dulbecco's modified Eagle's medium with $10 \% \mathrm{FBS}$ at $37^{\circ} \mathrm{C}$ in an incubator with $5 \% \mathrm{CO}_{2}$. Calcium phosphate reagent [in $250 \mathrm{ml}$ : 2D-Glucose (0.5 g), HEPES (2.5 g) KCl $(0.18 \mathrm{~g}) \mathrm{NaCl}$ $(4.0 \mathrm{~g})$ and $\left.\mathrm{Na}_{2} \mathrm{HPO}_{4}(0.05 \mathrm{~g})\right]$ was used for virus production, as previously described (13). Transduced positive $\mathrm{CD} 34^{+}$cells were selected with $1 \mu \mathrm{g} / \mathrm{ml}$ puromycin.

Flow cytometry analysis. The cells were collected following centrifugation ( $300 \mathrm{x} \mathrm{g}$ at room temperature for $5 \mathrm{~min}$ ), the supernatant was aspirated and the cells were washed twice with iced $2 \mathrm{ml}$ PBS containing 1\% FBS. The cells were then blocked with Human BD Fc Block ${ }^{\mathrm{TM}}$ (1:100) at room temperature for $30 \mathrm{~min}$. A total of $1 \mu \mathrm{l}$ anti-CD41 (cat. no. 559777) and $1 \mu 1$ anti-CD42 (cat. no. 555471) antibodies (both 1:100; BD Biosciences) was added to 1\% FBS in $100 \mu \mathrm{l}$
PBS, and incubated with the cells at room temperature for 30 min. The BD FACSDiva software and BD LSRFortessa ${ }^{\mathrm{TM}}$ were used for data collection, and FlowJo software was used for data analysis.

Reverse transcription-quantitative polymerase chain reaction (RT-qPCR). RT-qPCR was performed to measure the mRNA expression levels. Total RNA was isolated from all transfected and non-transfected CD34 ${ }^{+}$cells using an RNeasy Micro kit (cat. no. 74004; Qiagen GmbH, Hilden, Germany). Total RNA samples $(2 \mu \mathrm{g})$ were reverse transcribed using a SuperScript ${ }^{\circledR}$ First-Strand Synthesis System for RT-PCR (cat. no. 11904-018; Thermo Fisher Scientific, Inc.). qPCR was performed using a SYBR ${ }^{\circledR}$ Green Real-time PCR Master Mix-Plus kit (Thermo Fisher Scientific, Inc.). The RT-qPCR conditions were as follows: An initial denaturation step at $94^{\circ} \mathrm{C}$ for $10 \mathrm{~min}, 35$ cycles of $94^{\circ} \mathrm{C}$ for $30 \mathrm{sec}, 62^{\circ} \mathrm{C}$ for $30 \mathrm{sec}$ and $68^{\circ} \mathrm{C}$ for $1 \mathrm{~min}$, and a final extension step at $68^{\circ} \mathrm{C}$ for $7 \mathrm{~min}$. The samples were kept at $4^{\circ} \mathrm{C}$. The primer sequences used are listed in Table I. The copy number was normalized to GAPDH levels using the $2^{-\Delta \Delta \mathrm{Cq}}$ method (14).

Immunocytochemistry. Cells were fixed with $4 \%$ paraformaldehyde at room temperature for $1 \mathrm{~h}$ and permeabilized with $0.25 \%$ Triton X-100. The cells were then incubated with anti-PRMT1 (cat. no. 2449; Cell Signaling Technology, Inc., Danvers, MA, USA) and anti-RBM15 (cat. no. ab70549; Abcam, Cambridge, MA, USA) antibodies (both 1:100) for $2 \mathrm{~h}$ at room temperature. The cells were blocked with 5\% Bovine Serum Albumin (Sigma-Aldrich; Merck KGaA, Darmstadt, Germany) at room temperature for $30 \mathrm{~min}$. Following 1\% FBS in PBS washing, the cells were stained with AlexaFluor-488-conjugated goat anti-rabbit immunoglobulin G antibodies (1:1,000; cat. no. 14705; Cell Signaling Technology, Inc.) at room temperature for $30 \mathrm{~min}$ and counterstained with 4,6-diamidino-2-phenylindole at room temperature for $30 \mathrm{~min}$. Cell images were obtained at room temperature using confocal microscopy (Nikon AIR+; Nikon Corporation, Tokyo, Japan) with the magnification at $\mathrm{x} 400$.

Statistical analysis. Data are presented as mean \pm standard deviation from three independent experiments. Data was statistical analysis using GraphPad Prism software (version 6; GraphPad Software, Inc., La Jolla, CA, USA). One-way analysis of variance followed by the Student Newman-Keuls test was used to assess differences between multiple groups of data, and a Student's t-test was used to compare between two groups. $\mathrm{P}<0.05$ was considered to indicate a statistically significant difference.

\section{Results}

Upon TPO stimulation, human umbilical cord blood CD34 $4^{+}$ cells differentiate into mature MKs. To investigate the detailed role of PRMT1 and RBM15 in the process of megakaryocytopoiesis, $\mathrm{CD}^{+} 4^{+}$cells were isolated from human umbilical cord blood cells. These cells gave rise to $\mathrm{CD} 41^{+} \mathrm{CD} 42^{+}$when grown in medium containing TPO and SCF (Fig. 1A). The PRMT1 mRNA level decreased and RBM15 mRNA level increased during MK differentiation (Fig. 1B). On 
Table I. Primers used in reverse-transcription quantitative polymerase chain reaction.

\begin{tabular}{lll}
\hline Gene & Direction & Primer sequence $\left(5^{\prime}-3^{\prime}\right)$ \\
\hline Protein arginine methyltransferase 1 & Forward & CCAGTGGAGAAGGTGGACAT \\
& Reverse & CTCCCACCAGTGGATCTTGT \\
RNA binding motif protein 15 & Forward & TCCCACCTTGTGAGTTCTCC \\
& Reverse & GTCAGCGCCAAGTTTTCTCT \\
GAPDH & Forward & TGCACCACCAACTGCTTAGC \\
& Reverse & GGCATGGACTGTGGTCATGAG
\end{tabular}

A $(\%)$

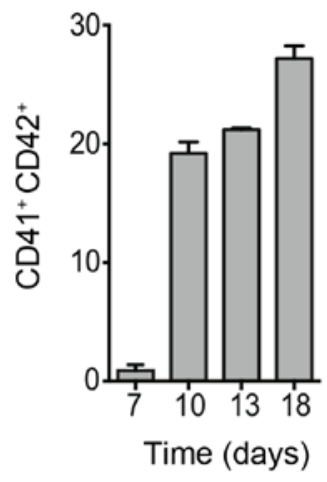

C

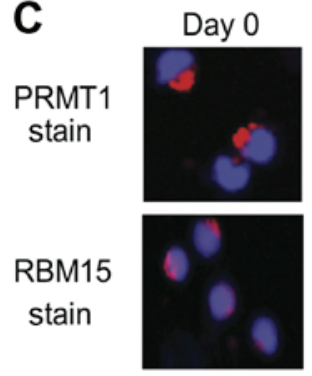

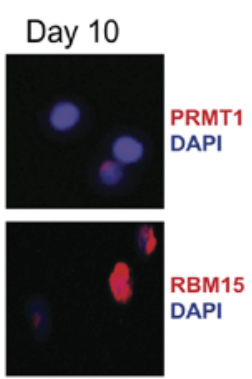

B

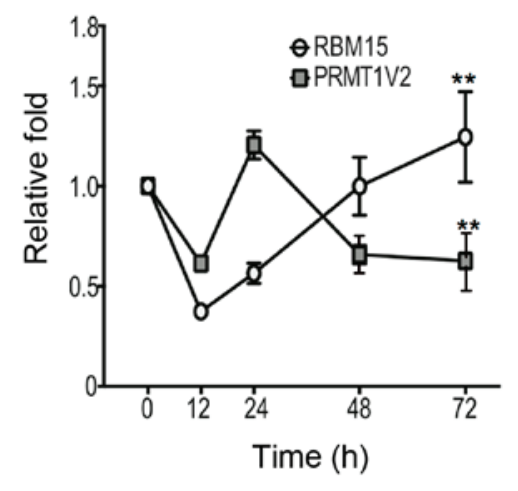

D

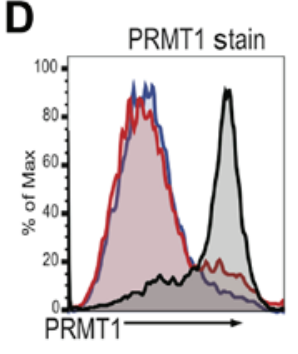

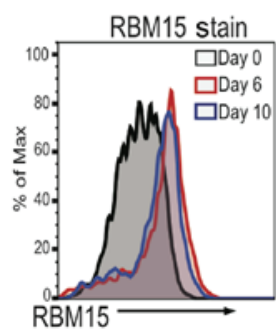

Figure 1. Upon TPO stimulation, human umbilical cord blood CD34+ cells differentiate into mature megakaryocytes. (A) FACS analysis of CD41 and CD42 double-positive cells cultured in medium containing high TPO on days 7, 10, 13 and 18. (B) Changes to RBM15 and PRMT1 expression at 12, 24, 48 and $72 \mathrm{~h}$ of TPO treatment. The mRNA levels were calculated as the mean \pm standard deviation from three independent experiments. (C) Intracellular immunostaining with anti-PRMT1 and anti-RBM15 antibodies (magnification, x400). (D) PRMT1 and RBM15 protein levels were detected by FACS analysis in the course of megakaryocyte differentiation. Results are displayed as histogram overlays. ${ }^{* * *} \mathrm{P}<0.01 \mathrm{vs} .0 \mathrm{~h}$. CD, cluster of differentiation; FACS, fluorescence-activated cell sorting; RBM15, RNA binding motif protein 15; PRMT1, protein arginine methyltransferase 1; PRMT1V2, PRMT1 V2 isoform; DAPI, 4,6-diamidino-2-phenylindole; TPO, thrombopoitin.

day 10 , intracellular immunostaining was conducted with anti-PRMT1 and anti-RBM15 antibodies. The results demonstrated that PRMT1-positive cells were markedly reduced at day 10 compared to day 0 , while RBM15-positive cells increased during MK differentiation (Fig. 1C). Consistently, it was demonstrated that intracellular PRMT1 protein level was markedly reduced at day 10 compared to day 0 according to FACS analysis, while the intracellular protein concentration of RBM15 was elevated (Fig. 1D). These data suggest that the PRMT1 and RBM15 protein levels are dynamically regulated during normal MK differentiation.

PRMT1 blocks the differentiation of human umbilical cord blood $C D 34^{+}$cells into mature MKs. To validate the role of PRMT1 in the process of megakaryocytopoiesis, CD $34^{+}$cells were transduced with lentivirus expressing PRMT1. FACS analysis demonstrated that, on day $10, \mathrm{CD} 41^{+} \mathrm{CD} 42^{+}$double-positive cells were markedly reduced when cells were cultured in high TPO medium (Fig. 2A). RT-qPCR analysis demonstrated that PRMT1 mRNA expression levels were significantly increased in $\mathrm{CD} 34^{+}$cells transfected with the vector or PRMT1 lentivirus and cultured in $\mathrm{CD}_{34}{ }^{+}$cells medium (Fig. 2B). However, the RBM15 mRNA level did not change significantly in cells transfected with the vector or PRMT1 lentivirus (Fig. 2C). FACS analysis of PRMT1-overexpressing CD $34^{+}$cells validated that the RBM15 protein level was markedly reduced compared with the level in the cells transfected with the control vector (Fig. 2D). Immunofluorescence microscopy with anti-RBM15 antibody staining further confirmed that the RBM15 protein level was reduced in PRMT1-overexpressing cells (Fig. 2E). 
A

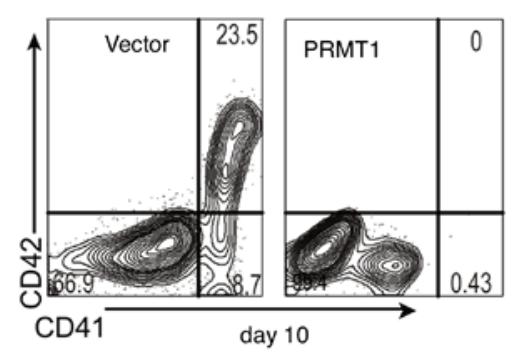

D

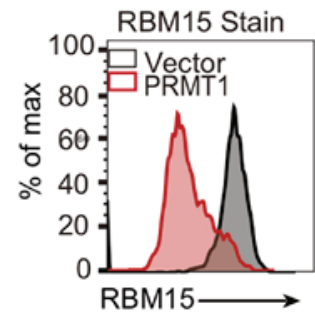

B

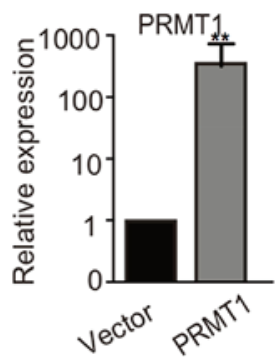

$\mathrm{E}$

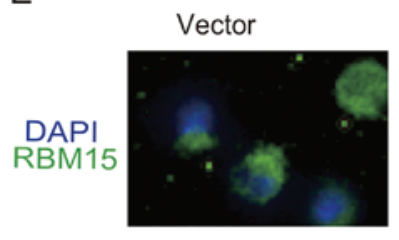

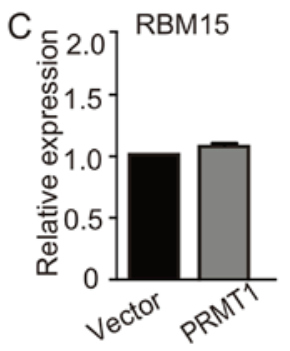

PRMT1

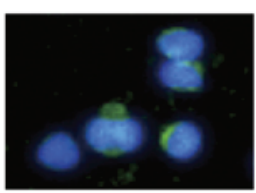

Figure 2. PRMT1 blocks the differentiation of human umbilical cord blood CD34 ${ }^{+}$cells into mature megakaryocytes. (A) FACS analysis of CD41 and CD42 double-positive cells cultured in high TPO-containing medium on day 10. (B) PRMT1 and (C) RBM15 mRNA expression levels in CD34 ${ }^{+}$cells overexpressing PRMT1. ${ }^{* *} \mathrm{P}<0.01$ vs. Vector. Three independent experiments were performed. (D) FACS analysis of the RBM15 protein expression level in cells with or without PRMT1 overexpression. (E) Intracellular immunostaining with anti-RBM15 antibody (magnification, x400). PRMT1, protein arginine methyltransferase 1; CD, cluster of differentiation; FACS, fluorescence-activated cell sorting; RBM15, RNA binding motif protein 15; DAPI, 4,6-diamidino-2-phenylindole; TPO, thrombopoitin.

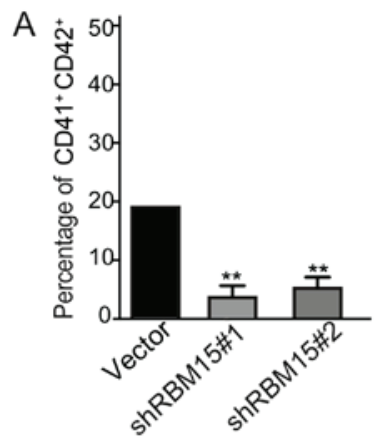

B

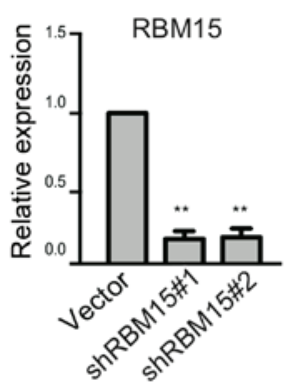

C

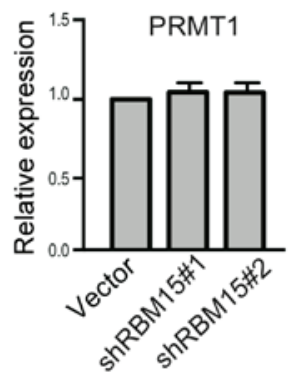

D

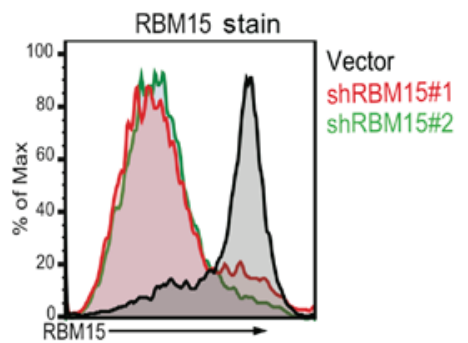

$\mathrm{E}$

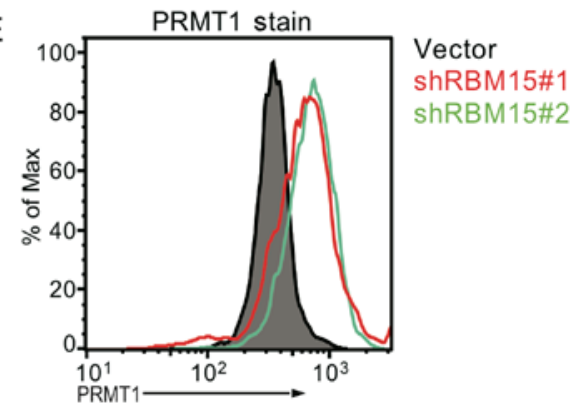

Figure 3. Knockdown of RBM15 blocks megakaryocytic differentiation. (A) Percentages of CD41 $1^{+} \mathrm{CD} 42^{+}$double-positive cells cultured in high TPO-containing medium on day 10 following RBM15 knock down. mRNA expression levels of (B) RBM15 and (C) PRMT1 in CD34 ${ }^{+}$cells with RBM15 knockdown. Three independent experiments were performed. (D) FACS analysis of the RBM15 protein level in cells with or without RBM15 knockdown. (E) Intracellular immunostaining with anti-PRMT1 antibody. ${ }^{* *} \mathrm{P}<0.01$ vs. the vector group. RBM15, RNA binding motif protein 15; CD, cluster of differentiation; FACS, fluorescence-activated cell sorting; PRMT1, protein arginine methyltransferase 1; sh, short hairpin; TPO, thrombopoitin.

These data suggest that PRMT1 blocks the differentiation of human umbilical cord blood $\mathrm{CD} 34^{+}$cells into mature MKs due to the decreased RBM15 protein level. RBM15 is important for MK differentiation.

Knockdown of RBM15 produces fewer mature MKs. To further validate the importance of RBM15 in regulating MK differentiation, RBM15 expression was knocked down using shRNA in human cord blood $\mathrm{CD} 34^{+}$cells and the cells were then cultured in MK differentiation medium. It was demonstrated that knocking down RBM15 caused a significant decrease in the $\mathrm{CD}_{4} 1^{+}$and $\mathrm{CD} 42^{+}$double-positive cells compared with those treated with the vector control (Fig. 3A). The RBM15 mRNA expression level was significantly decreased following 

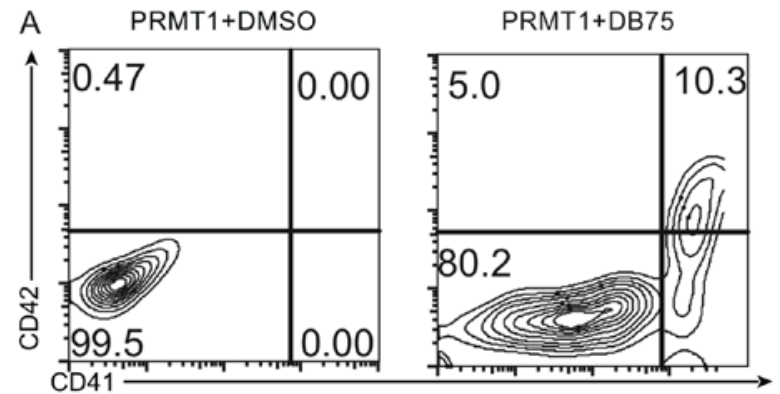

B

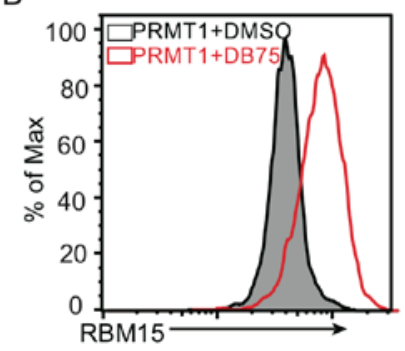

Figure 4. Treatment with PRMT1 inhibitor rescues PRMT1-blocked megakaryocytic differentiation. (A) FACS analysis of CD41 and CD42 expression following treatment with DB75. (B) FACS analysis of the RBM15 protein expression level in cells ectopically expressing PRMT1 with or without treatment with DB75. PRMT1, protein arginine methyltransferase 1; FACS, fluorescence-activated cell sorting; CD, cluster of differentiation; DB75, PRMT1 inhibitor; DMSO, dimethyl sulfoxide.

RBM15 knock down compared with the vector group, however, the PRMT1 mRNA expression level was not significantly affected (Fig. 3B and C). Flow cytometry demonstrated that the expression level of RBM15 was decreased (Fig. 3D) following knock down of RBM15, while PRMT1 increased (Fig. 3E). These data indicate that the PRMT1-RBM15 axis regulates megakaryocytic differentiation at the protein level, and the reduction of RBM15 expression produces fewer mature MKs.

PRMT1 inhibitor rescues PRMT1-blocked MK differentiation. To further investigate the function of PRMT1 in megakaryocytic differentiation, DB75 was used to treat the cells overexpressing PRMT1 protein and cultured in the MK differentiation medium. It was observed that the CD $41^{+} \mathrm{CD} 42^{+}$double-positive cells were increased following treatment with PRMT1 inhibitor compared to the number of double-positive cells observed following dimethyl sulfoxide (DMSO) treatment on day 10 (Fig. 4A). Additionally, the RBM15 protein level increased following treatment with PRMT1 inhibitor compared with that observed following DMSO treatment (Fig. 4B). These results demonstrate that PRMT1 expression affects MK differentiation via regulating the RBM15 protein level. These data provide a novel role of the PRMT1-RBM15 axis in regulating megakaryocytic differentiation.

To further investigate the function of PRMT1 in megakaryocytic differentiation, a PRMT1 inhibitor, DB75 (Sigma-Aldrich; Merck KGaA), was used to treat the cells overexpressing PRMT1 protein and cultured in the MK differentiation medium.

\section{Discussion}

Normal MK differentiation may be divided into two phases: From human stem cells (HSC) to MK progenitor cells and from MK progenitor cells to high polyploidy MK cells (15). Defective megakaryopoiesis is responsible for various types of blood disorders (1). Thus, MKs are regarded as a crucial source for treating thrombocytopenia, acute megakaryocytic leukemia (M7), myeloproliferative diseases and myelodysplasia syndrome, which affect a wide range of patients (2-4). Understanding the molecular mechanisms of megakaryocytic differentiation is important for developing novel therapies for hematopoietic malignance with defective MKs.
PRMT1 serves critical roles in various cellular processes, such as RNA splicing (16). PRMT1 is highly expressed in acute myeloid and lymphoid leukemia, as well as in solid tumors $(17,18)$. Therefore, PRMT1-mediated MK blockage may contribute to leukemogenesis. PRMT1 promotes the production of MK-erythroid progenitor cells; however, PRMT1 has to be turned off to generate mature, polyploidy $\mathrm{CD} 41^{+} \mathrm{CD} 42^{+} \mathrm{MK}$ cells (8). The present study demonstrated that PRMT1 negatively modulated differentiation toward the megakaryocytic lineage in human $\mathrm{CD} 34^{+}$hematopoietic cells. PRMT1 activity is downregulated during megakaryocytic differentiation. Immunostaining and flow cytometry analysis were used in the present study to demonstrate, for the first time (to the best of our knowledge), that the PRMT1 protein level is dynamically regulated during differentiation down to the MK lineage with primary cells. The dynamic regulation of PRMT1 concentration reciprocally regulates the RBM15 protein concentration. In the future, to identify upstream signals that are responsible for regulating PRMT1 activity, it is crucial to fully understand how hematopoiesis is regulated.

RBM15 is required to maintain the homeostasis of long-term hematopoietic stem cells and the differentiation of MK cells $(10,11)$. RBM15 protein stability is controlled by PRMT1 (5). RBM15 is involved in chromosome translocation $\mathrm{t}(1 ; 22)$, which produces the RBM15-megakaryoblastic leukemia 1 (MKL1) fusion protein associated with acute (A)MKL $(19,20)$. PRMT1 is upregulated in AMKL (21). Consistent with RBM15 knock down in human primary cells, RBM15 knockdown in mice produces a low percentage of mature MKs (22). Recently, the incidence of hematological malignancies has been increasing, which is problematic for many families (23). Therefore, novel methods for treatment are required. Targeting the RBM15/PRMT1 pathway may be a novel therapeutic approach for related hematological malignancies.

Dysregulation of the PRMT1-RBM15 pathway may be a common mechanism in hematological malignancies. The present study identified a novel role of PRMT1 in the negative regulation of human megakaryocytic differentiation via regulating the RBM15 protein level. DB75 is a PRMT1 inhibitor that has been demonstrated to kill some leukemia cells (24). The present study demonstrated that DB75 rescues PRMT1-blocked MK differentiation via promoting RBM15 expression. Our previous data indicated 
that PRMT1 methylates RBM15 at R578 and promotes RBM15 protein level degradation via ubiquitylation in a leukemia cell line (8). The present study also suggested that PRMT1 reduces RBM15 expression in human stem cells, which may contribute to defective megakaryopoiesis Therefore, targeting the RBM15/PRMT1 pathway may be a novel therapeutic approach for related hematological malignancies.

\section{Acknowledgements}

The present project was supported by grants from the Nature Science Foundation of China (NSFC; grant nos. 81270570 and 81470365) to YL and a fellowship from China Scholarship Council received by SJ.

\section{References}

1. Bourquin JP, Subramanian A, Langebrake C, Reinhardt D, Bernard O, Ballerini $\mathrm{P}$, Baruchel A, Cavé H, Dastugue N, Hasle H, et al: Identification of distinct molecular phenotypes in acute megakaryoblastic leukemia by gene expression profiling. Proc Natl Acad Sci USA 103: 3339-3344, 2006.

2. Je EM, Yoo NJ, Kim YJ, Kim MS and Lee SH: Mutational analysis of splicing machinery genes SF3B1, U2AF1 and SRSF2 in myelodysplasia and other common tumors. Int J Cancer 133 . 260-265, 2013.

3. Yamamoto S, Toyama D, Yatsuki H, Higashimoto K, Soejima $\mathrm{H}$ and Isoyama K: Acute megakaryocytic leukemia (AMKL,FAB;M7) with Beckwith-Wiedemann syndrome. Pediatr Blood Cancer 55: 733-735, 2010.

4. Han Y, Tang Y, Chen J, Liang J, Ye C, Ruan C and Wu D: Low-dose decitabine for patients with thrombocytopenia following allogeneic hematopoietic stem cell transplantation: A pilot therapeutic study. JAMA Oncol 1: 249-251, 2015.

5. Rhodes DR, Kalyana-Sundaram S, Mahavisno V, Varambally R, Yu J, Briggs BB, Barrette TR, Anstet MJ, Kincead-Beal C, Kulkarni P, et al: Oncomine 3.0: Genes, pathways, and networks in a collection of 18,000 cancer gene expression profiles. Neoplasia 9: 166-180, 2007.

6. Zolotukhin AS, Uranishi H, Lindtner S, Bear J, Pavlakis GN and Felber BK: Nuclear export factor RBM15 facilitates the access of DBP5 to mRNA. Nucleic Acids Res 37: 7151-7162, 2009.

7. Xiao N, Laha S, Das SP, Morlock K, Jesneck JL and Raffel GD: Ottl $(\mathrm{Rbm} 15)$ regulates thrombopoietin response in hematopoietic stem cells through alternative splicing of c-Mpl. Blood 125: 941-948, 2015.

8. Zhang L, Tran NT, Su H, Wang R, Lu Y, Tang H, Aoyagi S, Guo A, Khodadadi-Jamayran A, Zhou D, et al: Cross-talk between PRMT1-mediated methylation and ubiquitylation on RBM15 controls RNA splicing. eLife 4: pii: e07938, 2015.

9. Chang HC, Jones OW, Bradshaw C, Sarkar S and Porreco RP: Enhancement of human amniotic cell growth by Ficoll-Paque gradient fractionation. In Vitro 17: 81-90, 1981.

10. Yang Z, Augustin J, Chang C, Hu J, Shah K, Chang CW, Townes T and Jiang H: The DPY30 subunit in SET1/MLL complexes regulates the proliferation and differentiation of hematopoietic progenitor cells. Blood 124: 2025-2033, 2014.
11. Horiuchi K, Kawamura T, Iwanari H, Ohashi R, Naito M, Kodama T and Hamakubo T: Identification of Wilms' tumor 1 -associating protein complex and its role in alternative splicing and the cell cycle. J Biol Chem 288: 33292-33302, 2013.

12. Lee JH and Skalnik DG: Rbm15-Mkl1 interacts with the Setd1b histone H3-Lys4 methyltransferase via a SPOC domain that is required for cytokine-independent proliferation. PLoS One 7: e42965, 2012

13. Vu LP, Perna F, Wang L, Voza F, Figueroa ME, Tempst P, Erdjument-Bromage H, Gao R, Chen S, Paietta E, et al: PRMT4 blocks myeloid differentiation by assembling a methyl-RUNX1-dependent repressor complex. Cell Rep 5: $1625-1638,2013$.

14. Livak KJ and Schmittgen TD: Analysis of relative gene expression data using real-time quantitative PCR and the 2(-Delta Delta C(T)) method. Methods 25: 402-408, 2001.

15. Gao Y, Smith E, Ker E, Campbell P, Cheng EC, Zou S, Lin S, Wang L, Halene S and Krause DS: Role of RhoA-specific guanine exchange factors in regulation of endomitosis in megakaryocytes. Dev Cell 22: 573-584, 2012.

16. Bedford MT and Clarke SG: Protein arginine methylation in mammals: Who, what, and why. Mol Cell 33: 1-13, 2009.

17. Mathioudaki K, Papadokostopoulou A, Scorilas A, Xynopoulos D, Agnanti N and Talieri M: The PRMT1 gene expression pattern in colon cancer. Br J Cancer 99: 2094-2099, 2008.

18. Chang YI, Hua WK, Yao CL, Hwang SM, Hung YC, Kuan CJ, Leou JS and Lin WJ: Protein-arginine methyltransferase 1 suppresses megakaryocytic differentiation via modulation of the p38 MAPK pathway in K562 cells. J Biol Chem 285: 20595-20606, 2010.

19. Ma Z, Morris SW, Valentine V, Li M, Herbrick JA, Cui X, Bouman D, Li Y, Mehta PK, Nizetic D, et al: Fusion of two novel genes, RBM15 and MKL1, in the t(1;22) (p13; q13) of acute megakaryoblastic leukemia. Nat Genet 28: 220-221, 2001.

20. Mercher T, Coniat MB, Monni R, Mauchauffe M, Nguyen Khac F, Gressin L, Mugneret F, Leblanc T, Dastugue N, Berger $\mathrm{R}$ and Bernard OA: Involvement of a human gene related to the Drosophila spen gene in the recurrent $\mathrm{t}(1 ; 22)$ translocation of acute megakaryocytic leukemia. Proc Natl Acad Sci USA 98: 5776-5779, 2001.

21. Baldwin RM, Morettin A, Paris G, Goulet I and Côté J: Alternatively spliced protein arginine methyltransferase 1 isoform PRMT1v2 promotes the survival and invasiveness of breast cancer cells. Cell Cycle 11: 4597-4612, 2012.

22. Mercher T, RaffelGD,Moore SA, Cornejo MG, Baudry-Bluteau D, Cagnard N, Jesneck JL, Pikman Y, Cullen D, Williams IR, et al: The OTT-MAL fusion oncogene activates RBPJ-mediated transcription and induces acute megakaryoblastic leukemia in a knockin mouse model. J Clin Invest 119: 852-864, 2009.

23. Chihara D, Ito H, Matsuda T, Shibata A, Katsumi A, Nakamura S, Tomotaka S, Morton LM, Weisenburger DD and Matsuo K: Differences in incidence and trends of haematological malignancies in Japan and the United States. Br J Haematol 164: 536-545, 2014.

24. Yan L, Yan C, Qian K, Su H, Kofsky-Wofford SA, Lee WC, Zhao X, Ho MC, Ivanov I and Zheng YG: Diamidine compounds for selective inhibition of protein arginine methyltransferase 1 . J Med Chem 57: 2611-2622, 2014. 